Elsevier required licence: (C) <2018>. This manuscript version is made available under the CC-BY-NCND 4.0 license http://creativecommons.org/licenses/by-nc-nd/4.0/

The definitive publisher version is available online at

https://linkinghub.elsevier.com/retrieve/pii/S0020025516315493 


\title{
A reducibility method for the weak linear bilevel programming problems and a case study in principal-agent
}

\author{
Yue Zheng ${ }^{\mathrm{a}, \mathrm{b}}$, Guangquan Zhang ${ }^{\mathrm{b}}$, Zhen Zhang ${ }^{\mathrm{b}, \mathrm{c}}$, Jie Lu${ }^{\mathrm{b}}$ \\ ${ }^{\text {aS }}$ chool of Management, Huaibei Normal University, Huaibei 235000, P.R. China \\ ${ }^{\mathrm{b} D e c i s i o n}$ Systems and e-Service Intelligence Lab, Centre for Artificial Intelligence (CAI), \\ University of Technology Sydney, NSW 2007, Australia \\ 'Institute of Systems Engineering, Dalian University of Technology, Dalian 116024, P.R. China
}

\begin{abstract}
A weak linear bilevel programming (WLBP) problem often models problems involving hierarchy structure in expert and intelligent systems under the pessimistic point. In the paper, we deal with such a problem. Using the duality theory of linear programming, the WLBP problem is first equivalently transformed into a jointly constrained bilinear programming problem. Then, we show that the resolution of the jointly constrained bilinear programming problem is equivalent to the resolution of a disjoint bilinear programming problem under appropriate assumptions. This may give a possibility to solve the WLBP problem via a single-level disjoint bilinear programming problem. Furthermore, some examples illustrate the solution process and feasibility of the proposed method. Finally, the WLBP problem models a principal-agent problem under the pessimistic point that is also compared with a principal-agent problem under the optimistic point.
\end{abstract}

Keywords: Bilevel programming; Duality theory; Bilinear programming; Principal-agent

MSC: 90C26;90C30

\section{Introduction}

Bilevel programming problems are hierarchical optimization problems in which their constraints are defined in part by another parametric optimization problem, and have been investigated by many authors. The reader can refer to the monographs and surveys $[9,13,15,16,26,34,35,45,46]$.

Corresponding author, Jie Lu, E-mail: jie.lu@uts.edu.au 
As is well-known, bilevel programming plays an exceedingly important role in a variety of fields, such as transportation [10], electricity markets [44], supply chain [22], product distribution [39], economics [8], ecology [23] and engineering [25]. When the set of solutions of the lower level problem is not a singleton, however, it is difficult for the leaders to optimize his/her choice unless he knows the follower's reaction to his/her choice. In this situation, it is rational that the leader guards against the possible worst choice of the follower. This leads to a weak bilevel programming problem, see $[29,32,48]$ and the references therein.

The weak bilevel programming problem, also called a pessimistic formulation of bilevel programming or a weak Stackelberg problem [32,36], has been studied for decades. For example, Refs. [1-3,33] presented sufficient conditions for guaranteeing that solutions to such a problem exist; Refs. $[28,30,31,36]$ discussed the stability behavior of perturbed weak bilevel programming problems; and Refs. [14,17] dealt with optimality conditions. In addition, the weak bilevel programming model has been widely applied to various real-world problems, such as risk averse second best toll pricing [8], production planing [40] and principal-agent problem [40].

A limited number of papers have been contributed to solution algorithms for the weak bilevel programming problems. For example, Loridan and Morgan [32] presented an approximation method indirectly through a sequence of optimistic bilevel programming problems. Wiesemann et al. [43] presented a solvable $\varepsilon$-approximation algorithm for an independent pessimistic bilevel programming problem in which the feasible region of the lower level problem does not depend on the upper level decision variable. Cervinka et al. [11] proposed a new numerical method to compute approximate and relaxed pessimistic solutions to a mathematical program with equilibrium constraints which is a generalized bilevel programming problem.

Note that, weak bilevel programming problem even in its simplest form, i.e. in which both objective functions of leader and follower are linear and the constraint region is a polyhedron, is NP-hard [43]. Such a problem is called weak linear bilevel programming (WLBP) problem. There are two main methods to solve WLBP problems: penalty method and Kth-Best algorithm.

In a penalty method, a penalized problem is obtained by using the strong duality theory of linear programming to solve a WLBP problem. In fact, it is a disjoint bilinear programming problem (for details, see Section 2) but its objective function or constraint region depends on a penalty parameter $[1,47]$. In general, penalty parameters may be very large and lead to computational 
instabilities.

In the Kth-Best algorithm, the advantages are that such a algorithm can obtain a global solution of WLBP problems and terminates after the finite number of iterations [49]. However, the prior condition of its implement is generating all vertices of the constraint region of WLBP problems. It is not an easy work although there are many methods proposed to obtain all vertices of a polyhedron.

In this paper, we present a reducibility method for a WLBP problem which is different from the related papers $[1,47,49]$, and has the following features:

(i) in our paper, the WLBP problem is reduced to disjoint bilinear programming problems in which the objective function and the constraint region do not involve penalty parameters.

(ii) to obtain a solution of the WLBP problem, the proposed reducibility method is finite, that is, it only requires solving two disjoint bilinear programming problems and a linear programming problem.

(iii) the proposed method is only applied to solving the WLBP problem at present. That is, it depends on the structure of the targeted problem which shows a disadvantage of the proposed method.

The contributions of the paper are as follows.

(i) In fact, the WLBP problem is a three layered structural optimization problem. An advantage of the proposed reducibility method is that the WLBP problem is reduced to a single-level scalar optimization problem (i.e. disjoint bilinear programming problem) which is easier in the model structure, theoretical analysis and algorithm design than that of the WBLP problem.

(ii) We show that the resolution of the resulting jointly constrained bilinear programming problem via transforming the WLBP problem is equivalent to the resolution of a disjoint bilinear programming problem. This may give a possibility to solve indirectly the WLBP problem via a disjoint bilinear programming problem.

(iii) Indeed, the proposed method is a method for solving the disjoint bilinear programming problem. Other methods solve such a problem but depend on the penalty parameters, for example, in $[1,47]$. A comparison is made with these penalty methods in Section 5.2. It is demonstrated that in most cases the proposed method can obtain the same solutions as that of penalty methods in $[1,47]$. However, these penalty methods lead to computational instabilities when penalty 
parameters are very large.

(iv) To the author's knowledge, most studies ten years ago were focused on theory analysis of weak bilevel programming problems (e.g. existence results of solutions, stability behavior and optimality condition). Not so much has been discussed about the solution algorithms. The proposed reducibility method in this paper not only enriches the research WLBP problems, but also provides a way to study the weak bilevel programming problems with special structures.

The remainder of this paper is organized as follows. In the next section, we recall the bilinear programming and WLBP problems. In Section 3, we present a duality transformation model, and reduce the WLBP problem to a disjoint bilinear programming problem in Section 4. Section 5 presents some numerical examples to illustrate the proposed method. Section 6 develops an example of principal-agent problem to describe the application of the proposed method. Finally, concluding remarks are summarized in Section 7.

\section{Formulation and basic definitions}

In this section, we first review the bilinear programming problem in detail and then give the formulation and basic definitions of the WLBP problem.

\subsection{Bilinear programming problem}

In general, the bilinear programming problem is formulated as follows:

$$
\begin{aligned}
& \min _{\mathbf{x}, \mathbf{y}}\left[\mathbf{c}^{\cdot} \mathbf{x}+\mathbf{y}^{\bullet} \mathbf{Q} \mathbf{x}+\mathbf{d}^{\bullet} \mathbf{y}\right] \\
& \text { s.t. }(\mathbf{x}, \mathbf{y}) \in S:=\{(\mathbf{x}, \mathbf{y}): \mathbf{C x}+\mathbf{D y}, \mathbf{q}, \mathbf{x}, \mathbf{y} . . \mathbf{0}\},
\end{aligned}
$$

where $\mathbf{x}, \mathbf{c} \in i^{n}, \mathbf{y}, \mathbf{d} \in i^{m}, \mathbf{C} \in i^{p \times n}, \mathbf{D} \in i^{p \times m}, \mathbf{q} \in i^{p}$, and $\cdot$ stands for transpose.

In problem (1), the objective function is called bilinear if it reduces to a linear one by fixing the vector $\mathbf{x}$ or $\mathbf{y}$ to a particular value. The bilinear programming problem (1) is said to be jointly constrained bilinear programming problem $[5,24,41]$.

A number of authors have investigated special cases of problem (1). Most of the effort has focused on the following bilinear programming problem:

$$
\begin{aligned}
& \min _{\mathbf{x}, \mathbf{y}}\left[\mathbf{c}^{\bullet} \mathbf{x}+\mathbf{y}^{\bullet} \mathbf{Q x}+\mathbf{d}^{\cdot} \mathbf{y}\right] \\
& \text { s.t. } \mathbf{x} \in X, \mathbf{X} \in \boldsymbol{Y},
\end{aligned}
$$

where $\boldsymbol{X}$ and $\boldsymbol{Y}$ are given polyhedra. The variables $\mathbf{x}$ and $\mathbf{y}$ participating in the bilinear term of the 
objective function in problem (2) are independently constrained. Problem (2) is said to be disjoint bilinear programming problem [4,7]. It is also called separably constrained bilinear programming problem [41].

Problem (2) can be solved by the cutting plane method of Ritter [37]. Konno [27] modified this method to solve problem (2) but failed to guarantee convergence to a global solution. Very similar to that of [27], Vaish and Shetty [42] proposed a cutting plane method which has not guarantee of finite convergence. To avoid constructing expensive disjunctive facial cuts and achieve fast convergence, Ding and Al-Khayyal [18] presented two linear cutting plane method which combines the generation of polar cuts with the computation of lower bounds. Using the duality theory of linear programming, Falk [20] developed a finite branch-and-bound algorithm; Gallo and Ulkucu [21] presented a new variant of cutting plane method. Audet et al. [7] developed an exact method without any assumptions regarding boundedness of the feasible region or of the optimal objective value. Alarie et al. [4] proposed a new algorithm which combines concavity cuts and branch-and-bound for problem (2). More recently, Effati, Mansoori and Eshaghnezhad [19] applied the projection neural network to solving problem (2).

The reader interested in a more detailed overview of the different methods of problems (1) and (2) can be referred to the surveys [5,6].

\subsection{WLBP problem}

In this paper, we consider the following WLBP problem:

$$
\min _{\mathbf{x} \in X} \sup _{\mathbf{y} \in \Psi(\mathbf{x})}\left[\mathbf{c}^{\cdot} \mathbf{x}+\mathbf{d}_{\mathbf{1}}^{\mathbf{y}} \mathbf{y}\right]
$$

where $\Psi(\mathbf{x})$ is the set of solutions to the lower level problem

$$
\begin{aligned}
& \min _{\mathbf{y} . \mathbf{0}} d_{2} \mathbf{y} \\
& \text { s.t. } \mathbf{A x}+\mathbf{B y},, \mathbf{b} .
\end{aligned}
$$

Here, $\mathbf{x}, \mathbf{c} \in i^{n}, \mathbf{y}, \mathbf{d}_{1}, \mathbf{d}_{2} \in i^{m}, \mathbf{A} \in i^{p \times n}, \mathbf{B} \in i^{p \times m}, \mathbf{b} \in i^{p}$, and $\boldsymbol{X}$ is a closed subset of $i^{n}$.

Definition 2.1. (a) Constraint region of problem (3):

$$
W:=\{(\mathbf{x}, \mathbf{y}): \mathbf{x} \in \boldsymbol{X}, \mathbf{A x}+\mathbf{B y},, \mathbf{b}, \mathbf{y} . . \mathbf{0}\} .
$$

(b) Projection of $\boldsymbol{W}$ onto the leader's decision space:

$\boldsymbol{W}(\boldsymbol{X}):=\{\mathbf{x} \in \boldsymbol{X}: \exists \mathbf{y}$, such that $(\mathbf{x}, \mathbf{y}) \in \boldsymbol{W}\}$.

(c) Feasible set for the follower $\forall \mathbf{x} \in \boldsymbol{W}(\boldsymbol{X})$ : 


$$
\boldsymbol{Y}(\mathbf{x}):=\{\mathbf{y}: \mathbf{B y},, \mathbf{b}-\mathbf{A x}, \mathbf{y} . . \mathbf{0}\} .
$$

(d) The follower's rational reaction set for $\mathbf{x} \in \boldsymbol{W}(\boldsymbol{X})$ :

$$
\Psi(\mathbf{x}):=\left\{\mathbf{y}: \mathbf{y} \in \arg \min \left[\mathbf{d}_{2} \mathbf{y}: \mathbf{y} \in \boldsymbol{Y}(\mathbf{x})\right]\right\} .
$$

(e) Inducible region or feasible region of the leader:

$$
\operatorname{IR}:=\{(\mathbf{x}, \mathbf{y}):(\mathbf{x}, \mathbf{y}) \in \boldsymbol{W}, \mathbf{y} \in \Psi(\mathbf{x})\} .
$$

To introduce the concept of a solution to problem (3) (also called pessimistic solution), one usually employs the following value function $\varphi(\mathbf{x})$ :

$$
\varphi(\mathbf{x}):=\mathbf{c}^{\cdot} \mathbf{x}+\sup _{\mathbf{y} \in \Psi(\mathbf{x})} \mathbf{d}_{\mathbf{1}}^{\cdot} \mathbf{y} .
$$

Definition 2.2. A point $\left(\mathbf{x}^{*}, \mathbf{y}^{*}\right) \in \boldsymbol{I} \boldsymbol{R}$ is called a pessimistic solution to problem (3), if

$$
\begin{aligned}
& \varphi\left(\mathbf{x}^{*}\right)=\mathbf{c}^{\cdot} \mathbf{x}^{*}+\mathbf{d}_{1} \mathbf{y}^{*}, \\
& \varphi\left(\mathbf{x}^{*}\right), \varphi(\mathbf{x}), \forall(\mathbf{x}, \mathbf{y}) \in \mathbb{I R} .
\end{aligned}
$$

\section{Duality transformation}

In this section, using the duality theory of linear programming, we will transform the WLBP problem into an equivalent single-level nonconvex optimization problem. To ensure the existence of solutions of the WLBP problem, we first introduce the following two assumptions which are from [1]:

(A1) For any $\mathbf{x} \in \boldsymbol{X}, \boldsymbol{Y}(\mathbf{x}) \neq \varnothing$, and there exists a compact subset $\boldsymbol{Z}$ of $i^{m}$ such that $\boldsymbol{Y}(\mathbf{x}) \subset \boldsymbol{Z}$.

(A2) The set $\boldsymbol{X}$ is a nonempty polytope.

Then, we have the following result.

Theorem 3.1. Under assumptions (A1) and (A2), the WLBP problem has at least one solution.

Proof. It follows immediately from Theorem 3.3 in [1] or Theorem 4.1 in [33].

Obviously, the dual problem of (4) is written as:

$$
\begin{gathered}
\max _{\mathbf{z} . \mathbf{0}}-(\mathbf{b}-\mathbf{A x})^{\bullet} \mathbf{z} \\
\text { s.t. }-\mathbf{B}^{\cdot} \mathbf{z}, \mathbf{d}_{2}, \\
\text { where } z \in i^{p} .
\end{gathered}
$$


Denote the feasible region by $Z_{1}:=\left\{\mathbf{z} . . \mathbf{0}:-\mathbf{B}^{\cdot} \mathbf{z}, \mathbf{d}_{2}\right\}$, and the duality gap between problems (4) and (5) by $\pi(\mathbf{x}, \mathbf{y}, \mathbf{z}):=\mathbf{d}_{2}^{\cdot} \mathbf{y}+(\mathbf{b}-\mathbf{A x})^{\bullet} \mathbf{z}$.

By the strong duality theorem of linear programming, we have $\mathbf{y} \in \Psi(\mathbf{x})$ if and only if $\mathbf{y} \in \boldsymbol{Y}(\mathbf{x})$ and there exists $\mathbf{z} \in \boldsymbol{Z}_{1}$ such that $\pi(\mathbf{x}, \mathbf{y}, \mathbf{z})=0$.

Since $\pi(\mathbf{x}, \mathbf{y}, \mathbf{z}) . .0$ is always true, $\pi(\mathbf{x}, \mathbf{y}, \mathbf{z})=0$ can be replaced by $\pi(\mathbf{x}, \mathbf{y}, \mathbf{z}),, 0$.

Based on the above results, we find that $\max _{\mathbf{y} \in \Psi(\mathbf{x})} \mathbf{d}_{\mathbf{1}} \mathbf{y}$

is equivalent to the following problem:

$$
\begin{gathered}
\boldsymbol{P}(\mathbf{x}) \quad \max _{\mathbf{y}, \mathbf{z}} \mathbf{d}_{1} \mathbf{y} \\
\text { s.t. } \mathbf{d}_{2} \mathbf{y}+(\mathbf{b}-\mathbf{A x})^{\bullet} \mathbf{z},, 0, \\
\mathbf{B y},, \mathbf{b}-\mathbf{A x}, \\
-\mathbf{B}^{\cdot} \mathbf{z},, \mathbf{d}_{2}, \\
\mathbf{y}, \mathbf{z} . \mathbf{0} .
\end{gathered}
$$

Denote

$$
Z_{2}(\mathbf{x}):=\left\{(\mathbf{y}, \mathbf{z}): \mathbf{d}_{2}^{\bullet} \mathbf{y}+(\mathbf{b}-\mathbf{A x})^{\bullet} \mathbf{z},, 0, \mathbf{B y},, \mathbf{b}-\mathbf{A x},-\mathbf{B}^{\bullet} \mathbf{z},, \mathbf{d}_{2}, \mathbf{y}, \mathbf{z} . . \mathbf{0}\right\}
$$

Moreover, we denote by $\boldsymbol{V}(\mathrm{A})$ the set of vertices of a polyhedron A .

For each $\mathbf{x} \in \boldsymbol{X}$, the dual problem of (6) is:

$$
\begin{gathered}
\min _{u, v, w}(\mathbf{b}-\mathbf{A x})^{\bullet} \mathbf{v}+\mathbf{d}_{2}^{\cdot} \mathbf{w} \\
\text { s.t. }-\mathbf{d}_{2} \boldsymbol{u}-\mathbf{B}^{\cdot} \mathbf{v},,-\mathbf{d}_{1}, \\
-(\mathbf{b}-\mathbf{A x}) \boldsymbol{u}+\mathbf{B} \mathbf{w}, \mathbf{0}, \\
\boldsymbol{u}, \mathbf{v}, \mathbf{w} . . \mathbf{0},
\end{gathered}
$$

where $\boldsymbol{u} \in \mathbf{i}, \quad \mathbf{v} \in i^{p}$ and $\mathbf{w} \in i^{m}$.

Since both problems (6) and (7) have the same optimal value, the WLBP problem can be equivalently transformed into a jointly constrained bilinear programming problem as follows:

$$
\min _{\mathbf{x}, u, \mathbf{v}, \mathbf{w}} \mathbf{c}^{\cdot} \mathbf{x}+(\mathbf{b}-\mathbf{A x})^{\bullet} \mathbf{v}+\mathbf{d}_{\mathbf{2}}^{\cdot} \mathbf{w}
$$


s.t. $-\mathbf{d}_{2} \boldsymbol{u}-\mathbf{B}^{\cdot} \mathbf{v},-\mathbf{d}_{1}$,

$$
\begin{aligned}
& -(\mathbf{b}-\mathbf{A x}) \boldsymbol{u}+\mathbf{B w},, \mathbf{0}, \\
& \mathbf{x} \in \boldsymbol{X}, \boldsymbol{u}, \mathbf{v}, \mathbf{w} . . \mathbf{0} .
\end{aligned}
$$

Clearly, the feasible region of problem (8) has a bilinear inequality which may lead to computational complexity. So, in the following, we try to avoid this bilinear term.

\section{Reduction of (8) to a single-level disjoint bilinear programming problem}

In this section, exploiting the particular form of the nonconvex optimization problem (8), we will show that the resolution of the problem (8) is equivalent to the resolution of the following disjoint bilinear programming problems (9) and (10).

Consider the following two disjoint bilinear programming problems:

$$
\begin{gathered}
\min _{\mathbf{x}, u, \mathbf{v}, \mathbf{w}} \mathbf{c}^{\cdot} \mathbf{x}+(\mathbf{b}-\mathbf{A x})^{\cdot} \mathbf{v}+\mathbf{d}_{2}^{\cdot} \mathbf{w} \\
\text { s.t. }-\mathbf{B}^{\cdot} \mathbf{v},,-\mathbf{d}_{1}, \\
\mathbf{B w}, \mathbf{0}, \\
\boldsymbol{u}=0, \\
\mathbf{x} \in \boldsymbol{X}, \mathbf{v}, \mathbf{w} . . \mathbf{0}
\end{gathered}
$$

and

$$
\begin{aligned}
& \min _{\mathbf{x}, u, v, \boldsymbol{w}} \mathbf{c}^{\cdot} \mathbf{x}+(\mathbf{b}-\mathbf{A x})^{\bullet} \mathbf{v}+u \mathbf{d}_{2} \boldsymbol{w}^{\boldsymbol{u}} \\
& \text { s.t. }-\mathbf{d}_{2} \boldsymbol{u}-\mathbf{B}^{\cdot} \mathbf{v},,-\mathbf{d}_{1} \text {, } \\
& \mathbf{A x}+\mathbf{B} \text {, }, \mathbf{b}, \\
& \mathbf{x} \in X, u . .0, \mathbf{v}, \mathbf{u} . \mathbf{0} .
\end{aligned}
$$

Before giving the relationships among problems (8)-(10), we first present some notations and lemmas. Denote the feasible regions of problems (8)-(10) respectively by

$$
\begin{aligned}
& \Omega:=\left\{(\mathbf{x}, \boldsymbol{u}, \mathbf{v}, \mathbf{w}):-\mathbf{d}_{2} \boldsymbol{u}-\mathbf{B}^{\cdot} \mathbf{v},,-\mathbf{d}_{1},-(\mathbf{b}-\mathbf{A x}) \boldsymbol{u}+\mathbf{B w},, \mathbf{0}, \mathbf{x} \in X, \boldsymbol{u} . .0, \mathbf{v}, \mathbf{w} . . \mathbf{0}\right\}, \\
& D:=\left\{(\mathbf{x}, \boldsymbol{u}, \mathbf{v}, \mathbf{w}):-\mathbf{B}^{\cdot} \mathbf{v},,-\mathbf{d}_{1}, \mathbf{B w}, \boldsymbol{0}, \boldsymbol{u}=0, \mathbf{x} \in X, \mathbf{v}, \mathbf{w} . . \mathbf{0}\right\}, \\
& \mathrm{E}:=\left\{\left(\mathbf{x}, \boldsymbol{u}, \mathbf{v}, \mathbf{W}^{\prime}\right):-\mathbf{d}_{2} u-\mathbf{B}^{\cdot} \mathbf{v},,-\mathbf{d}_{1}, \mathbf{A x}+\mathbf{B},, \mathbf{b}, \mathbf{x} \in X, \boldsymbol{u} . .0, \mathbf{v}, \ldots . .0\right\} .
\end{aligned}
$$


Furthermore, we split $\Omega$ into two sets:

$$
\begin{aligned}
& \Omega_{1}:=\{(\mathbf{x}, \boldsymbol{u}, \mathbf{v}, \mathbf{w}):(\mathbf{x}, \boldsymbol{u}, \mathbf{v}, \mathbf{w}) \in \Omega, \boldsymbol{u}=0\}, \\
& \Omega_{2}:=\{(\mathbf{x}, \boldsymbol{u}, \mathbf{v}, \mathbf{w}):(\mathbf{x}, \boldsymbol{u}, \mathbf{v}, \mathbf{w}) \in \Omega, \boldsymbol{u}>0\} .
\end{aligned}
$$

It is easy to verify that $\Omega_{1}=\boldsymbol{D}$.

$$
\begin{aligned}
& \text { Also, we split E into two sets: } \\
& \boldsymbol{E}_{1}:=\{(\mathbf{x}, \boldsymbol{u}, \mathbf{v}, \boldsymbol{W}):(\mathbf{x}, \boldsymbol{u}, \mathbf{v}, \boldsymbol{W}) \in \boldsymbol{E}, \boldsymbol{u}=0\}, \\
& \boldsymbol{E}_{2}:=\{(\mathbf{x}, \boldsymbol{u}, \mathbf{v}, \boldsymbol{W}):(\mathbf{x}, \boldsymbol{u}, \mathbf{v}, \boldsymbol{W}) \in \boldsymbol{E}, \boldsymbol{u}>0\} .
\end{aligned}
$$

The following three lemmas give the relationships among the feasible regions of problems (8)-(10).

Lemma 4.1. There is a one-one correspondence between $\Omega_{2}$ and $\boldsymbol{E}_{2}$.

Proof. It is easy to verify that there is a one-one correspondence between $\Omega_{2}$ and $\boldsymbol{E}_{2}$ by

$$
\begin{aligned}
& (\mathbf{x}, \boldsymbol{u}, \mathbf{v}, \mathbf{w}) \in \Omega_{2} \rightarrow\left(\mathbf{x}, \boldsymbol{u}, \mathbf{v}, \frac{\mathbf{w}}{\boldsymbol{u}}\right) \in \boldsymbol{E}_{2}, \\
& (\mathbf{x}, \boldsymbol{u}, \mathbf{v}, \boldsymbol{w}) \in \boldsymbol{E}_{2} \rightarrow(\mathbf{x}, \boldsymbol{u}, \mathbf{v}, \boldsymbol{u} \boldsymbol{w}) \in \Omega_{2} .
\end{aligned}
$$

This completes the proof.

Lemma 4.2. $\Omega=D \cup\left\{(\mathbf{x}, \boldsymbol{u}, \mathbf{v}, \boldsymbol{u} W): \forall(\mathbf{x}, \boldsymbol{u}, \mathbf{v}, \boldsymbol{W}) \in \boldsymbol{E}_{2}\right\}$.

Proof. The result follows immediately from Lemma 4.1.

Lemma 4.3. If assumptions (A1) and (A2) are satisfied, then $E \neq \varnothing$.

Proof. For each $\mathbf{x} \in \boldsymbol{X}$, it is easy to check that $\mathrm{Z}_{2}(\mathbf{x}) \neq \varnothing$ under assumption (A1). Then, it follows from Theorem 1 in [12] that $\Omega \neq \varnothing$.

Now, we prove $\boldsymbol{E} \neq \varnothing$ by contradiction. Suppose that $\mathrm{E}=\varnothing$, then $\boldsymbol{E}_{2}=\varnothing$ and $\Omega_{2}=\varnothing$. Furthermore, the set

$$
Q:=\left\{\mathbf{v}:-\mathbf{B}^{\cdot} \mathbf{v},-\mathbf{d}_{1}, \mathbf{v} \ldots \mathbf{0}\right\}
$$

is also empty, which implies that $\boldsymbol{D}=\varnothing$.

Thus, we have

$$
\Omega=\Omega_{1} \cup \Omega_{2}=D \cup \Omega_{2}=\varnothing
$$


which contradicts $\Omega \neq \varnothing$.

Hence, $\mathrm{E} \neq \varnothing$. This completes the proof.

The following two lemmas can guarantee the existence of solutions of problems (9) and (10) respectively.

Lemma 4.4. Under assumptions (A1) and (A2), if $\boldsymbol{D} \neq \varnothing$, then problem (9) has at least one solution in $\boldsymbol{V}(\boldsymbol{D})$.

Proof. For simplicity, we denote

$Z_{3}:=\left\{(\mathbf{v}, \mathbf{w}):-\mathbf{B}^{\bullet} \mathbf{v},,-\mathbf{d}_{1}, \mathbf{B w},, \mathbf{0}, \mathbf{v}, \mathbf{w} . . \mathbf{0}\right\}$

For each $\mathbf{x} \in \boldsymbol{X}$, consider the following linear programming problem:

$$
\min _{(\mathbf{v}, \mathbf{w}) \in Z_{3}}\left[(\mathbf{b}-\mathbf{A x})^{\cdot} \mathbf{v}+\mathbf{d}_{2} \mathbf{w}\right]
$$

and its dual problem is:

$$
\begin{gathered}
\max _{\mathbf{y}, \mathbf{z}} \mathbf{d}_{\mathbf{1}} \mathbf{y} \\
\text { s.t. } \mathbf{B y},, \mathbf{b}-\mathbf{A x}, \\
-\mathbf{B}^{\cdot} \mathbf{z}, \mathbf{d}_{2}, \\
\mathbf{y}, \mathbf{z} . . \mathbf{0} .
\end{gathered}
$$

Let $\theta_{1}(\bullet)$ be the marginal function defined on $X$ by

$$
\theta_{1}(\mathbf{x}):=\inf _{(\mathbf{v}, \mathbf{w}) \in \mathbf{Z}_{3}}\left[\mathbf{c}^{\cdot} \mathbf{x}+(\mathbf{b}-\mathbf{A x})^{\cdot} \mathbf{v}+\mathbf{d}_{2}^{\cdot} \mathbf{w}\right]
$$

It is easy to check that $\theta_{1}(\bullet)$ is a concave function, and we have

$$
\begin{aligned}
& \inf _{\mathbf{x} \in X} \theta_{1}(\mathbf{x}) \\
= & \inf _{\mathbf{x} \in X} \inf _{(\mathbf{v}, \mathbf{w}) \in Z_{3}}\left[\mathbf{c}^{\cdot} \mathbf{x}+(\mathbf{b}-\mathbf{A x})^{\cdot} \mathbf{v}+\mathbf{d}_{\mathbf{2}} \mathbf{w}\right] \\
= & \inf _{\mathbf{x} \in X}\left\{\mathbf{c}^{\cdot} \mathbf{x}+\sup _{(\mathbf{y}, \mathbf{z}) \in Y(\mathbf{x}) \times \mathbf{Z}_{1}} \mathbf{d}_{\mathbf{1}} \mathbf{y}\right\} \\
\ldots & \inf _{\mathbf{x} \in X, \mathbf{y} \in \mathbf{Y}(\mathbf{x})}\left[\mathbf{c}^{\cdot} \mathbf{x}+\mathbf{d}_{\mathbf{1}}^{\cdot} \mathbf{y}\right] .
\end{aligned}
$$

It follows from assumptions (A1) and (A2) that

$$
\inf _{\mathbf{x} \in X, \mathbf{y} \in \mathbf{Y}(\mathbf{x})}\left[\mathbf{c}^{\cdot} \mathbf{x}+\mathbf{d}_{1}^{\mathbf{y}} \mathbf{y}\right]
$$

is attained, and denote the optimal value by $\boldsymbol{F}^{*}$. Thus, the objective function $\theta_{1}(\mathbf{x})$ of problem 
(15) is bounded from below (e.g. $\boldsymbol{F}^{*}$ ). Using the result of Corollary 32.3.4 in [38], we can obtain that problem (15) has at least one solution in $\boldsymbol{V}(\boldsymbol{X})$.

Without loss of generality, suppose that $\mathbf{x}^{*}$ is a solution to problem (15). Substituting $\mathbf{x}$ with $\mathbf{x}^{*}$ in problem 13), we have

$$
\min _{(\mathbf{v}, \mathbf{w}) \in Z_{3}}\left[\left(\mathbf{b}-\mathbf{A} \mathbf{x}^{*}\right)^{\cdot} \mathbf{v}+\mathbf{d}_{\mathbf{2}}^{\cdot} \mathbf{w}\right] .
$$

Note that, the feasible region of problem (16) is not empty because of the result of Theorem 1 in [12] and the fact that the feasible region of problem (14) is not empty.

Moreover, the objective function of problem (16) is bounded from below (e.g. $\boldsymbol{F}^{*}-\mathbf{c}^{\cdot} \mathbf{x}^{*}$ ). Thus, there exists a solution $\left(\mathbf{v}^{*}, \mathbf{w}^{*}\right) \in \boldsymbol{V}\left(\boldsymbol{Z}_{3}\right)$ to the linear programming problem (16).

Hence, problem (9) has at least one solution $\left(\mathbf{x}^{*}, 0, \mathbf{v}^{*}, \mathbf{w}^{*}\right)$ in $\boldsymbol{V}(\boldsymbol{D})$. This completes the proof.

Lemma 4.5. Under assumptions (A1) and (A2), problem (10) has at least one solution in $\boldsymbol{V}(\mathrm{E})$.

Proof. Firstly, we introduce some notations:

$$
\begin{aligned}
& \boldsymbol{Z}_{4}:=\left\{(\boldsymbol{u}, \mathbf{v}):-\mathbf{d}_{2} \boldsymbol{u}-\mathbf{B}^{\cdot} \mathbf{v},,-\mathbf{d}_{1}, \boldsymbol{u} . .0, \mathbf{v} . . \mathbf{0}\right\}, \\
& \boldsymbol{Z}_{5}:=\{(\mathbf{x}, \boldsymbol{W}): \mathbf{A x}+\mathbf{B}, \boldsymbol{b}, \mathbf{x} \in \boldsymbol{X}, \boldsymbol{W} . \mathbf{0}\} .
\end{aligned}
$$

For each $\left(\mathbf{x}, \boldsymbol{Z}_{5}\right.$, consider the following linear programming problem:

$$
\min _{(u, \mathbf{v}) \in Z_{4}}\left[(\mathbf{b}-\mathbf{A x})^{\bullet} \mathbf{v}+\boldsymbol{u} \mathbf{d}_{2} \mathbf{w}\right]
$$

and its dual problem is:

$$
\begin{gathered}
\max _{\mathbf{y}} d_{1} \mathbf{y} \\
\text { s.t. } \mathbf{B y},, \mathbf{b}-\mathbf{A x}, \\
\mathbf{d}_{2} \mathbf{y}_{,}, \mathbf{d}_{2} \boldsymbol{w}, \\
\mathbf{y} . .0
\end{gathered}
$$

Denote the feasible region of problem (17) by $\$(\mathbf{x}, \mathbf{x})$.

Let $\theta_{2}(\bullet)$ be the marginal function defined on $Z_{5}$ by

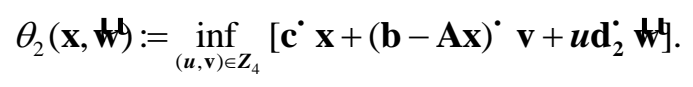


Similar to the second part of the proof of Lemma 4.4, we can prove that problem (10) has at least one solution in $\boldsymbol{V}(\mathrm{E})$.

Now, we have the following result which shows the relationships among problems (8)-(10).

Theorem 4.1. Let assumptions (A1) and (A2) be satisfied. If $\left(\mathbf{x}^{\prime}, \boldsymbol{u}^{\prime}, \mathbf{v}^{\prime}, \boldsymbol{w}^{\prime}\right)$ solves problem (10), then either $\left(\mathbf{x}^{\prime}, \boldsymbol{u}^{\prime}, \mathbf{v}^{\prime}, \boldsymbol{u}^{\prime} \boldsymbol{w}^{\prime}\right)$ is a solution to problem (8) or problem (8) is solved by a solution to problem (9).

Proof. To prove this result, we consider the following two cases:

Case 1: $\boldsymbol{u}^{\prime}=0$.

Since $\left(\mathbf{x}^{\prime}, \boldsymbol{u}^{\prime}, \mathbf{v}^{\prime}, \boldsymbol{w}^{\prime}\right)$ is a solution to problem (10), we have

$$
\begin{aligned}
\mathbf{c}^{\cdot} \mathbf{x}^{\prime}+\left(\mathbf{b}-\mathbf{A} \mathbf{x}^{\prime}\right)^{\cdot} \mathbf{v}^{\prime} & =\mathbf{c}^{\cdot} \mathbf{x}^{\prime}+\left(\mathbf{b}-\mathbf{A x} \mathbf{x}^{\prime}\right)^{\cdot} \mathbf{v}^{\prime}+\boldsymbol{u}^{\prime} \mathbf{d}_{2} \boldsymbol{w}^{\prime} \\
& , \mathbf{c}^{\cdot} \mathbf{x}+(\mathbf{b}-\mathbf{A x})^{\cdot} \mathbf{v}+u \mathbf{d}_{2}^{\prime} \boldsymbol{w}, \forall(\mathbf{x}, \boldsymbol{u}, \mathbf{v}, \boldsymbol{w}) \in E_{2} .
\end{aligned}
$$

Using the relationships between $\Omega_{2}$ and $\boldsymbol{E}_{2}$, i.e. (11) and (12), $\forall(\mathbf{x}, \boldsymbol{u}, \mathbf{v}, \mathbf{w}) \in \Omega_{2}$, we have

$$
\mathbf{c}^{\cdot} \mathbf{x}^{\prime}+\left(\mathbf{b}-\mathbf{A x ^ { \prime }}\right)^{\cdot} \mathbf{v}^{\prime}, \mathbf{c}^{\cdot} \mathbf{x}+(\mathbf{b}-\mathbf{A x})^{\bullet} \mathbf{v}+\mathbf{d}_{2}^{\prime} \mathbf{w}
$$

It is easy to check that $\left(\mathbf{x}^{\prime}, 0, \mathbf{v}^{\prime}, \mathbf{0}\right)$ is a feasible point of problem (9). Thus, $\boldsymbol{D} \neq \varnothing$, and it follows from Lemma 4.4 that problem (9) has at least one solution. Without loss of generality, assume that $\left(\mathbf{x}^{*}, \boldsymbol{u}^{*}, \mathbf{v}^{*}, \mathbf{w}^{*}\right)$ is a solution to problem (9). Then, we have

$$
\mathbf{c}^{\cdot} \mathbf{x}^{*}+\left(\mathbf{b}-\mathbf{A} \mathbf{x}^{*}\right)^{\cdot} \mathbf{v}^{*}+\mathbf{d}_{2} \mathbf{w}^{*}, \mathbf{c}^{\cdot} \mathbf{x}^{\prime}+\left(\mathbf{b}-\mathbf{A} \mathbf{x}^{\prime}\right)^{\cdot} \mathbf{v}^{\prime}
$$

Combining (18) and (19), $\forall(\mathbf{x}, \boldsymbol{u}, \mathbf{v}, \mathbf{w}) \in \Omega$, we can obtain that

$$
\mathbf{c}^{\cdot} \mathbf{x}^{*}+\left(\mathbf{b}-\mathbf{A} \mathbf{x}^{*}\right)^{\cdot} \mathbf{v}^{*}+\mathbf{d}_{2}^{*} \mathbf{w}^{*}, \mathbf{c}^{\cdot} \mathbf{x}+(\mathbf{b}-\mathbf{A x})^{\cdot} \mathbf{v}+\mathbf{d}_{2} \mathbf{w}
$$

which implies that $\left(\mathbf{x}^{*}, \boldsymbol{u}^{*}, \mathbf{v}^{*}, \mathbf{w}^{*}\right)$ is also a solution to problem (8).

Case 2: $u^{\prime}>0$.

$\forall\left(\mathbf{x}, \boldsymbol{u}, \mathbf{v}, \boldsymbol{W}^{\prime}\right) \in \boldsymbol{E}_{2}$, it follows from the definition of $\left(\mathbf{x}^{\prime}, \boldsymbol{u}^{\prime}, \mathbf{v}^{\prime}, \boldsymbol{w}^{\prime}\right)$ that

$$
c^{\cdot} x^{\prime}+\left(b-A x^{\prime}\right)^{\cdot} \mathbf{v}^{\prime}+u^{\prime} d_{2}^{\prime} w^{\prime}, c^{\cdot} x+(b-A x)^{\cdot} \mathbf{v}+u d_{2}^{\cdot} w^{\prime}
$$

Using the relationships between $\Omega_{2}$ and $\boldsymbol{E}_{2}$, i.e. (11) and (12), $\forall(\mathbf{x}, \boldsymbol{u}, \mathbf{v}, \mathbf{w}) \in \Omega_{2}$, we have 
$\mathbf{c}^{\cdot} \mathbf{x}^{\prime}+\left(\mathbf{b}-\mathbf{A} \mathbf{x}^{\prime}\right)^{\cdot} \mathbf{v}^{\prime}+\mathbf{d}_{2}^{\prime}\left(u^{\prime} w^{\prime}\right), \mathbf{c}^{\cdot} \mathbf{x}+(\mathbf{b}-\mathbf{A x})^{\bullet} \mathbf{v}+\mathbf{d}_{2}^{\prime} \mathbf{w}$.

Since we cannot determine whether the set $\Omega_{1}$ is empty, we consider the following two cases:

Case 2.1: $\Omega_{1}=\varnothing$. It follows from (20) that $\left(\mathbf{x}^{\prime}, \boldsymbol{u}^{\prime}, \mathbf{v}^{\prime}, \boldsymbol{u}^{\prime} \boldsymbol{w}^{\prime}\right)$ is a solution to problem (8).

Case 2.2: $\Omega_{1} \neq \varnothing$. Then we have $\boldsymbol{D} \neq \varnothing$, and assume that $\left(\mathbf{x}^{*}, \boldsymbol{u}^{*}, \mathbf{v}^{*}, \mathbf{w}^{*}\right)$ is a solution to problem (9). Furthermore, $\forall(\mathbf{x}, \boldsymbol{u}, \mathbf{v}, \mathbf{w}) \in \Omega_{1}$, we find that

$$
\mathbf{c}^{\cdot} \mathbf{x}^{*}+\left(\mathbf{b}-\mathbf{A} \mathbf{x}^{*}\right)^{\cdot} \mathbf{v}^{*}+\mathbf{d}_{2} \mathbf{w}^{*}, \mathbf{c}^{\cdot} \mathbf{x}+(\mathbf{b}-\mathbf{A x})^{\cdot} \mathbf{v}+\mathbf{d}_{2}^{*} \mathbf{w}
$$

Eqs. (20) and (21) yield that problem (8) has at least a solution $\left(\mathbf{x}_{0}^{0}, \%\right.$ from the set $S:=\left\{\left(\mathbf{x}^{\prime}, \boldsymbol{u}^{\prime}, \mathbf{v}^{\prime}, \boldsymbol{u}^{\prime} \boldsymbol{w}^{\prime}\right),\left(\mathbf{x}^{*}, \boldsymbol{u}^{*}, \mathbf{v}^{*}, \mathbf{w}^{*}\right)\right\}$ such that

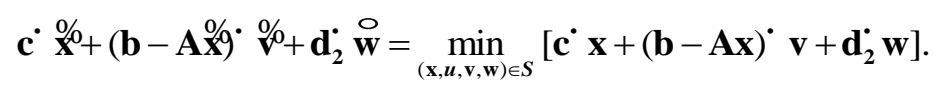

This completes the proof.

Furthermore, it follows from Theorem 4.1 and its proof that we can easily obtain the following result.

Theorem 4.2. Under assumptions (A1) and (A2), let $\left(\mathbf{x}^{*}, \boldsymbol{u}^{*}, \mathbf{v}^{*}, \mathbf{w}^{*}\right)$ (if it exists) and $\left(\mathbf{x}^{\prime}, \boldsymbol{u}^{\prime}, \mathbf{v}^{\prime}, w^{\prime}\right)$ be solutions to problems (9) and (10) respectively. Then, the resolution of problem (8) is equivalent to the two disjoint bilinear programming problems (9) and (10) in the sense that

1) If $\boldsymbol{u}^{\prime}=0$, then $\left(\mathbf{x}^{*}, \boldsymbol{u}^{*}, \mathbf{v}^{*}, \mathbf{w}^{*}\right)$ is a solution to problem (8).

2) If $\boldsymbol{u}^{\prime}>0$ and the solution of problem (9) does not exist, then $\left(\mathbf{x}^{\prime}, \boldsymbol{u}^{\prime}, \mathbf{v}^{\prime}, \boldsymbol{u}^{\prime} \boldsymbol{w}^{\prime}\right)$ is a solution to problem (8).

3) If $\boldsymbol{u}^{\prime}>0$ and problem (9) has a solution, then $\left(\mathbf{x}^{\circ}, \boldsymbol{v}^{\circ}, \mathbf{w}\right) \in \boldsymbol{S}$ satisfying (22) is a solution to problem (8).

Consequently, the resolution of problem (8) is equivalent to the resolution of the disjoint bilinear programming problems (9) and (10). Therefore, the WLBP problem is reduced to a disjoint bilinear programming problem. 


\section{Experiment results}

In this section, we provide two examples to illustrate solution process and feasibility of the proposed method.

Example 1:

$\min _{\mathbf{x} \in X} \max _{\mathbf{y} \in \Psi(\mathbf{x})}-8 \boldsymbol{x}_{1}-10 \boldsymbol{x}_{2}-2 \boldsymbol{y}_{1}+\boldsymbol{y}_{2}$

where $\boldsymbol{X}:=\left\{\mathbf{x}:=\left(\boldsymbol{x}_{1}, \boldsymbol{x}_{2}\right)^{*}: \boldsymbol{x}_{1}+\boldsymbol{x}_{2}, 10, \boldsymbol{x}_{1}, \boldsymbol{x}_{2} . .0\right\}$, and $\Psi(\mathbf{x})$ is the set of solutions to the lower level problem,

$\min _{\mathbf{y}}-\boldsymbol{y}_{1}-\boldsymbol{y}_{2}$

s.t. $\boldsymbol{y}_{1}+\boldsymbol{y}_{2}, 20+\boldsymbol{x}_{1}-\boldsymbol{x}_{2}$,

$\mathbf{y}:=\left(\boldsymbol{y}_{1}, \boldsymbol{y}_{2}\right)^{\bullet} \ldots \mathbf{0}$.

Example 2:

$\min _{\mathbf{x} \in X} \max _{\mathbf{y} \in \Psi(\mathbf{x})}-8 x_{1}-6 x_{2}-25 y_{1}-30 y_{2}+2 y_{3}+16 y_{4}$

where $X:=\left\{\mathbf{x}:=\left(x_{1}, x_{2}\right)^{*}: x_{1}+x_{2}, 10, x_{1}, x_{2} . .0\right\}$, and $\Psi(\mathbf{x})$ is the set of solutions of the lower level problem,

$$
\begin{gathered}
\min _{\mathbf{y}}-10 \boldsymbol{y}_{1}-10 \boldsymbol{y}_{2}-10 \boldsymbol{y}_{3}-10 \boldsymbol{y}_{4} \\
\text { s.t. } \boldsymbol{y}_{1}+\boldsymbol{y}_{2}+\boldsymbol{y}_{3}+\boldsymbol{y}_{4}, 10-\boldsymbol{x}_{1}-\boldsymbol{x}_{2}, \\
-\boldsymbol{y}_{1}+\boldsymbol{y}_{4}, 0.8 \boldsymbol{x}_{1}+0.8 \boldsymbol{x}_{2}, \\
\boldsymbol{y}_{2}+\boldsymbol{y}_{4}, 4 \boldsymbol{x}_{2}, \\
\mathbf{y}:=\left(\boldsymbol{y}_{1}, \boldsymbol{y}_{2}, \boldsymbol{y}_{3}, \boldsymbol{y}_{4}\right)^{\circ} . . \mathbf{0} .
\end{gathered}
$$

\subsection{Solution process of Example 1}

To better illustrate the proposed method in this paper, we explain definitions and theorems within the content of Example 1, and present a detailed solution process as follows.

For Example 1, the constraint region is:

$W:=\left\{(\mathbf{x}, \mathbf{y}): x_{1}+x_{2}, 10, y_{1}+y_{2}, 20+x_{1}-x_{2}, x_{1}, x_{2}, y_{1}, y_{2} . .0\right\}$

Projection of $\boldsymbol{W}$ onto the leader's decision space: 
$\boldsymbol{W}(\boldsymbol{X}):=\{\mathbf{x} \in \boldsymbol{X}: \exists \mathbf{y}$, such that $(\mathbf{x}, \mathbf{y}) \in \boldsymbol{W}\}$

The feasible set for the follower is:

$\boldsymbol{Y}(\mathbf{x}):=\left\{\mathbf{y}: \boldsymbol{y}_{1}+\boldsymbol{y}_{2}, 20+\boldsymbol{x}_{1}-\boldsymbol{x}_{2}, \boldsymbol{y}_{1}, \boldsymbol{y}_{2} . .0\right\}$

The follower's rational reaction set for $\mathbf{x} \in \boldsymbol{W}(\boldsymbol{X})$ :

$\Psi(\mathbf{x}):=\left\{\mathbf{y}: \boldsymbol{y}_{1}+\boldsymbol{y}_{2}=20+\boldsymbol{x}_{1}-\boldsymbol{x}_{2}, \boldsymbol{y}_{1}, \boldsymbol{y}_{2} . .0\right\}$.

Inducible region or feasible region of the leader:

$\mathrm{IR}:=\left\{(\mathbf{x}, \mathbf{y}): x_{1}+x_{2}, 10, y_{1}+y_{2}=20+x_{1}-x_{2}, x_{1}, x_{2}, y_{1}, y_{2} . .0\right\}$

In fact, this example has a unique solution $\left(\frac{0}{\mathbf{x}}, \mathbf{y}\right):=(0,10,0,10)$. Let us verify this by using our method. Using problem (9), this example can be transformed as follows:

$$
\begin{array}{cl}
\min _{x_{1}, x_{2}, u, v, w_{1}, w_{2}} & -8 x_{1}-10 x_{2}+\left(20+x_{1}-x_{2}\right) v-w_{1}-w_{2} \\
\text { s.t. } \quad & v, 2, \\
& -v,,-1, \\
& w_{1}+w_{2}, 0, \\
& u=0, \\
& x_{1}+x_{2}, 10, \\
& x_{1}, x_{2}, v, w_{1}, w_{2} . .0,
\end{array}
$$

and a solution to problem (23) is

$$
\left(\mathbf{x}^{*}, u^{*}, \mathbf{v}^{*}, \mathbf{w}^{*}\right):=\left(x_{1}^{*}, \boldsymbol{x}_{2}^{*}, \boldsymbol{u}^{*}, \boldsymbol{v}^{*}, \boldsymbol{w}_{1}^{*}, \boldsymbol{w}_{2}^{*}\right)=(0,10,0,1,0,0) \text {. }
$$

On the other hand, this example can be written as follows via problem (10):

$$
\begin{array}{cl}
\min _{\boldsymbol{x}_{1}, x_{2}, u, v, \hat{w}_{1}, \hat{w}_{2}} & -8 x_{1}-10 x_{2}+\left(20+x_{1}-x_{2}\right) v-u \hat{w}_{1}-u \hat{w}_{2} \\
\text { s.t. } \quad & u-v, 2, \\
& u-v,,-1, \\
& -x_{1}+x_{2}+\hat{w}_{1}+\hat{w}_{2}, 20 \\
& x_{1}+x_{2}, 10 \\
& x_{1}, x_{2}, u, v, \hat{w}_{1}, \hat{w}_{2} . .0 .
\end{array}
$$


A solution to problem (24) is

$\left(\mathbf{x}^{\prime}, u^{\prime}, \mathbf{v}^{\prime}, w^{\prime}\right):=\left(x_{1}^{\prime}, x_{2}^{\prime}, u^{\prime}, v^{\prime}, \hat{w}_{1}^{\prime}, \hat{w}_{2}^{\prime}\right)=(0,10,0,1,0,0)$

It follows from the first result in Theorem 4.2 that

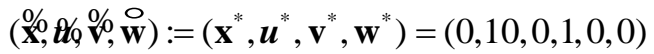

is a solution to problem (8) since $\boldsymbol{u}^{\prime}=0$.

Furthermore, we solve the following linear programming problem $\boldsymbol{P}\left(\mathbf{x}^{\%}\right)$ :

$$
\begin{gathered}
\min _{\boldsymbol{y}_{1}, \boldsymbol{y}_{2}, z}-2 \boldsymbol{y}_{1}+\boldsymbol{y}_{2} \\
\text { s.t. }-\boldsymbol{y}_{1}-\boldsymbol{y}_{2}+10 \boldsymbol{z}_{,}, 0, \\
\boldsymbol{y}_{1}+\boldsymbol{y}_{2}, 10, \\
-\boldsymbol{z}_{\boldsymbol{\prime}},-1, \\
\boldsymbol{y}_{1}, \boldsymbol{y}_{2}, \boldsymbol{z} . .0,
\end{gathered}
$$

and a solution is $\left(\frac{\mathscr{y}}{0}, \mathbf{z}\right)=(0,10,1)$.

Therefore, $(\mathbf{x}, \mathbf{y})=(0,10,0,10)$ is a solution to Example 1, and the leader's optimal objective function value is -90 .

Similar as above, we can obtain that $\mathbf{x}^{\circ}:=(10,0)$ with $\mathbf{y}^{\circ}:=(0,0,0,0)$ is a solution of Example 2 whose optimal objective function value of the leader is -80 .

\subsection{Comparison results}

Using penalty method and duality theory of linear programming, Aboussoror and Mansouri [1] transformed WLBP into a disjoint bilinear programming problem with a penalty parameter. Very similar to that of [1], Zheng et al. [47] presented another disjoint bilinear programming problem. However, in our paper, the WLBP problem is reduced to two disjoint bilinear programming problems without penalty parameters. In this section, so we describe some comparison results for these disjoint bilinear programming problems.

To solve Example 2, using problems (9) and (10), we consider the following two disjoint bilinear programming problems:

$$
\min _{x_{1}, x_{2}, u, v_{1}, v_{2}, v_{3}, w_{1}, w_{2}, w_{3}, w_{4}}-6 x_{1}-8 x_{2}+\left(10-x_{1}-x_{2}\right) v_{1}+\left(0.8 x_{1}+0.8 x_{2}\right) v_{2}
$$




$$
+4 x_{2} v_{3}-10\left(w_{1}+w_{2}+w_{3}+w_{4}\right)
$$

$$
\begin{array}{ll}
\text { s.t. } & \boldsymbol{x}_{1}+\boldsymbol{x}_{2}, 10, \\
& -\boldsymbol{v}_{1}+\boldsymbol{v}_{2}, 25, \\
& -\boldsymbol{v}_{1}-\boldsymbol{v}_{3}, 30, \\
& -\boldsymbol{v}_{1},-2, \\
& -\boldsymbol{v}_{1}-\boldsymbol{v}_{2}-\boldsymbol{v}_{3},-16, \\
& \boldsymbol{u}=0, \\
& \boldsymbol{w}_{1}+\boldsymbol{w}_{2}+\boldsymbol{w}_{3}+\boldsymbol{w}_{4}, 0, \\
- & \boldsymbol{w}_{1}+\boldsymbol{w}_{4}, 0, \\
\boldsymbol{w}_{2}+\boldsymbol{w}_{4}, 0, \\
\boldsymbol{x}_{1}, \boldsymbol{x}_{2}, \boldsymbol{v}_{1}, \boldsymbol{v}_{2}, \boldsymbol{v}_{3}, \boldsymbol{w}_{1}, \boldsymbol{w}_{2}, \boldsymbol{w}_{3}, \boldsymbol{w}_{4} . .0,
\end{array}
$$

and

$$
\begin{array}{ll}
\min _{x_{1}, x_{2}, u, v_{1}, v_{2}, v_{3}, \hat{w}_{1}, \hat{w}_{2}, \hat{w}_{3}, \hat{w}_{4}} & -6 x_{1}-8 x_{2}+\left(10-x_{1}-x_{2}\right) v_{1}+\left(0.8 x_{1}+0.8 x_{2}\right) v_{2} \\
& +4 x_{2} v_{3}-10 u\left(\hat{w}_{1}+\hat{w}_{2}+\hat{w}_{3}+\hat{w}_{4}\right) \\
\text { s.t. } \quad & x_{1}+x_{2}, 10, \\
& 10 u-v_{1}+v_{2}, 25, \\
& 10 u-v_{1}-v_{3}, 30, \\
& 10 u-v_{1},-2, \\
& 10 u-v_{1}-v_{2}-v_{3},-16, \\
& \hat{w}_{1}+\hat{w}_{2}+\hat{w}_{3}+\hat{w}_{4}+x_{1}+x_{2}, 10, \\
& -0.8 x_{1}-0.8 x_{2}-\hat{w}_{1}+\hat{w}_{4}, 0, \\
& -4 x_{2}+\hat{w}_{2}+\hat{w}_{4}, 0, \\
& x_{1}, x_{2}, u, v_{1}, v_{2}, v_{3}, \hat{w}_{1}, \hat{w}_{2}, \hat{w}_{3}, \hat{w}_{4} . .0 .
\end{array}
$$


Table 1 Comparison results for Example 1

\begin{tabular}{lllll}
\hline Method & & $\boldsymbol{x}^{*}$ & $f^{*}$ & Time(s) \\
\hline The proposed method & Problem (23) & $(0,10)$ & -90 & 0.001 \\
& Problem (24) & $(0,10)$ & -90 & 0.001 \\
Penalty method in [1] & $k=1$ & $(0,10)$ & -90 & 0.001 \\
& $k=10$ & $(0,10)$ & -90 & 0.001 \\
& $k=100$ & $(0,10)$ & -90 & 0.001 \\
& $k=1000$ & $(0,10)$ & -90 & 0.001 \\
Penalty method in [47] & $k=1$ & $(0,10)$ & -90 & 0.001 \\
& $k=10$ & $(0,10)$ & -90 & 0.001 \\
& $k=100$ & $(0,10)$ & -90 & 0.001 \\
& $k=1000$ & $(0,10)$ & -90 & 0.001 \\
\hline
\end{tabular}

Table 2 Comparison results for Example 2

\begin{tabular}{lllll}
\hline Method & & $\boldsymbol{x}^{*}$ & $\boldsymbol{f}^{*}$ & Time(s) \\
\hline The proposed method & Problem (25) & $(10,0)$ & -80 & 0.001 \\
& Problem (26) & $(10,0)$ & -80 & 0.001 \\
Penalty method in [1] & $k=1$ & $(10,0)$ & -80 & 0.001 \\
& $k=10$ & $(10,0)$ & -80 & 0.001 \\
& $k=100$ & $(10,0)$ & -80 & 0.001 \\
& $k=1000$ & $(10,0)$ & -80 & 0.001 \\
Penalty method in [47] & $k=1$ & $(10,0)$ & -80 & 0.001 \\
& $k=10$ & $(10,0)$ & -80 & 0.001 \\
& $k=100$ & $(10,0)$ & -80 & 0.001 \\
& $k=1000$ & $(10,0)$ & -80 & 0.001 \\
\hline
\end{tabular}

In our experiments, the numerical tests were run on a PC $(1.3 \mathrm{GHz}$ Inter Core i5, 4GB 1600 MHz DDR3). All disjoint bilinear programming problems are solved by NEOS GAMS/SCIP (for details, see https://neos-server.org/neos/solvers/go:scip/GAMS.html). SCIP is one of the recently 
updated solvers on the NEOS server which is commonly used for the global solution of nonlinear programming problems.

We first solve problems (23)-(26), and report the numerical results in Tables 1 and 2 where $\boldsymbol{x}^{*}$, $f^{*}$ and Time(s) are projection of solutions onto the leader's decision space, the leader's optimal objective function value and execution time of the associated problems respectively. Furthermore, for comparison purposes, we respectively choose penalty parameters $k=1,10,100$ and 1000 to solve the resulting disjoint bilinear programming problems in $[1,47]$. The results of these penalty methods are given in Tables 1 and 2 as well.

As shown in Tables 1 and 2, the solutions of the proposed method are the same as that of the methods developed in $[1,47]$. So is the execution time. Unfortunately, when the penalty parameters are increased to very large (e.g. $10^{9}$ in Examples 1 and 2), these penalty methods lead to computational instabilities. Therefore, we consider the proposed reducibility method as a feasible and an effective approach for solving WLBP problems.

\section{A case-based example and analysis}

In this section, a case study on a principal-agent (PA) problem is presented to demonstrate the applicability of the proposed model and method. Note that, it is not the first time that weak (pessimistic) bilevel optimization is applied to the PA problem. As far as known, Tsoukalas, Wiesemann and Rustem [40] first present a PA problem based on the pessimistic bilevel programming. The problem analyzed in their paper is an independent pessimistic bilevel problem. In other words, the feasible region of the agent does not depend on the decision variables of the principal. In our paper, a class of PA problem where the feasible region of the agent depends on the decision variables of the principal is considered.

\subsection{An example}

In game theory, a PA problem is one in which the principal delegates a task to the agent in exchange for a wage. The PA problem can be described as follows. Suppose that the principal determines a contract $\mathbf{x}$. The agent then selects an effort or an action $\mathbf{y}$ to perform the task for the principal. The agent selects a $\mathbf{y}$ to maximize his/her utility function $\boldsymbol{A}(\mathbf{y})$, and the principal wishes to maximize his/her utility function $\boldsymbol{P}(\mathbf{x}, \mathbf{y})$. 
In the following, assume that $\mathbf{x}=\left(\boldsymbol{x}_{1}, \boldsymbol{x}_{2}\right)^{\bullet}, \mathbf{x} \in \boldsymbol{X}=\left\{\mathbf{x}: \boldsymbol{x}_{1}+\boldsymbol{x}_{2}, 6, \boldsymbol{x}_{1}, \boldsymbol{x}_{2} . .0\right\}, \quad \mathbf{y}=\left(\boldsymbol{y}_{1}, \boldsymbol{y}_{2}\right)^{\circ}$ and $\mathbf{y} \in \boldsymbol{Y}(\mathbf{x})=\left\{\mathbf{y}: \boldsymbol{y}_{1}+\boldsymbol{y}_{2}, 12-\boldsymbol{x}_{1}-2 \boldsymbol{x}_{2}, \boldsymbol{y}_{1}, \boldsymbol{y}_{2} . .0\right\}$. The utility function of the agent is determined by $\boldsymbol{A}(\mathbf{y})=\boldsymbol{y}_{1}+\boldsymbol{y}_{2}$, and the utility function of the principal generated by the contract $\mathbf{x}$ and the effort $\mathbf{y}$ is determined by $\boldsymbol{P}(\mathbf{x}, \mathbf{y})=5 \boldsymbol{x}_{1}+8 \boldsymbol{x}_{2}+4 \boldsymbol{y}_{1}-\boldsymbol{y}_{2}$.

In general, the principal cannot observe $\mathbf{y}$. When designing a contract, he may be risk averse (pessimistic) or risk prone (optimistic).

With the risk prone principal, an optimistic PA (OPA) problem can be written as follows:

$$
\begin{aligned}
& \max _{\mathbf{x}, \mathbf{y}} 5 \boldsymbol{x}_{1}+8 \boldsymbol{x}_{2}+4 \boldsymbol{y}_{1}-\boldsymbol{y}_{2} \\
& \text { s.t. } \mathbf{x} \in \boldsymbol{X}=\left\{\mathbf{x}: \boldsymbol{x}_{1}+\boldsymbol{x}_{2}, 6, \boldsymbol{x}_{1}, \boldsymbol{x}_{2} . .0\right\}, \\
& \max _{\mathbf{y}} \boldsymbol{y}_{1}+\boldsymbol{y}_{2} \\
& \text { s.t. } \boldsymbol{y}_{1}+\boldsymbol{y}_{2}, 12-\boldsymbol{x}_{1}-2 \boldsymbol{x}_{2}, \\
& \boldsymbol{y}_{1}, \boldsymbol{y}_{2} . .0
\end{aligned}
$$

Note that OPA problem is an optimistic bilevel programming problem [15].

With the risk averse principal, a pessimistic PA (PPA) problem can be described as follows:

$$
\begin{aligned}
& \max _{\mathbf{x}} \min _{\mathbf{y}}\left[5 x_{1}+8 x_{2}+4 y_{1}-y_{2}\right] \\
& \text { s.t. } \mathbf{x} \in \boldsymbol{X}=\left\{\mathbf{x}: \boldsymbol{x}_{1}+\boldsymbol{x}_{2}, 6, \boldsymbol{x}_{1}, \boldsymbol{x}_{2} . .0\right\}, \\
& \max _{\mathbf{y}} \boldsymbol{y}_{1}+\boldsymbol{y}_{2} \\
& \text { s.t. } \boldsymbol{y}_{1}+\boldsymbol{y}_{2}, 12-\boldsymbol{x}_{1}-2 \boldsymbol{x}_{2}, \\
& \boldsymbol{y}_{1}, \boldsymbol{y}_{2} \ldots 0 .
\end{aligned}
$$

In fact, PPA problem is a WLBP problem discussed in this paper.

\subsection{Results analysis}

Many existing methods (e.g. penalty function method, Kth-Best algorithm and branch-and-bound algorithm) can solve the OPA problem. It has a global solution $\left(\mathbf{x}_{o}, \mathbf{y}_{o}\right)=(6,0,9,0)$ and the associated objective function value of the principal is $\boldsymbol{P}_{\boldsymbol{o}}=66$. Note 
that the agent's rational reaction set with $\mathbf{x}=\mathbf{x}_{o}=(6,0)$ is

$$
\Psi\left(\mathbf{x}_{\boldsymbol{o}}\right)=\left\{\left(\boldsymbol{y}_{1}, \boldsymbol{y}_{2}\right): \boldsymbol{y}_{1}+\boldsymbol{y}_{2}=9, \boldsymbol{y}_{1}, \boldsymbol{y}_{2} . .0\right\}
$$

Clearly, the element of the set $\Psi\left(\mathbf{x}_{o}\right)$ is not unique. As $\mathbf{y}$ varies in $\Psi\left(\mathbf{x}_{o}\right)$, the objective function values of the principal vary from 66 to 21 . It is easy to check that the objective function value of the principal is equal to $\hat{\boldsymbol{P}}_{\boldsymbol{o}}=21$ when $\mathbf{y}=(0,9) \in \Psi\left(\mathbf{x}_{\boldsymbol{o}}\right)$. Here $\hat{\boldsymbol{P}}_{\boldsymbol{o}}=21 \mathrm{can}$ be referred to as the value of the worst case by the OPA method with $\mathbf{x}=(6,0)$.

Using the proposed method in this paper, we can solve the PPA problem whose solution is $\left(\mathbf{x}_{p}, \mathbf{y}_{p}\right)=(0,6,0,3)$ and the associated objective function value of the principal is $\boldsymbol{P}_{p}=45$. This value is less than that of the OPA model (i.e. 66) and is greater than that of the worst case by the OPA method (i.e. 21). Note that the agent's rational reaction set with $\mathbf{x}=\mathbf{x}_{p}=(0,6)$ is

$$
\Psi\left(\mathbf{x}_{\boldsymbol{p}}\right)=\left\{\left(\boldsymbol{y}_{1}, \boldsymbol{y}_{2}\right): \boldsymbol{y}_{1}+\boldsymbol{y}_{2}=3, \boldsymbol{y}_{1}, \boldsymbol{y}_{2} . .0\right\}
$$

Clearly, the element of the set $\Psi\left(\mathbf{x}_{p}\right)$ is not unique. As $\mathbf{y}$ varies in $\Psi\left(\mathbf{x}_{p}\right)$, the objective function values of the principal vary from 60 to 45 . It is easy to very that the objective function value of the principal is $\hat{\boldsymbol{P}}_{p}=60$ when $\mathbf{y}=(3,0) \in \Psi\left(\mathbf{x}_{p}\right)$. Here $\hat{\boldsymbol{P}}_{\boldsymbol{p}}=60$ can be referred to as the value of the best case by the PPA model with $\mathbf{x}=(0,6)$.

To further compare the performance of the OPA and the PPA methods, we compute the average and variation of the principal's objective function value for a given contract. Here the average is defined as the average between the best and worst cases, and the variation as the difference between the best and worst cases of the principal's objective function values. Clearly, the average value of the PPA method (i.e. 52.5) is greater than that of the OPA method (i.e. 43.5). Moreover, the variation for the OPA method is $\left|\boldsymbol{P}_{\boldsymbol{o}}-\hat{\boldsymbol{P}}_{\boldsymbol{o}}\right|=45$ which is higher than that of the PPA method $\left(\left|\boldsymbol{P}_{p}-\hat{\boldsymbol{P}}_{p}\right|=15\right)$. This suggests $\mathbf{x}=(0,6)$ generates the agent's rational reaction set whose average of the principal's objective function value is greater than that by $\mathbf{x}=(0,6)$ and with a smaller variation. In other words, a contract designed by the risk averse principal is superior to that by the risk prone principal at least for this example.

As shown in the above results analysis, using the pessimistic (weak) bilevel programming to 
model the PA problem is of practical significance. Therefore, researches on how to solve weak bilevel programming problem is still attractive.

\section{Conclusions and further study}

In this paper, we consider a weak linear bilevel programming (WLBP) problem which is NP-hard. Using the duality theory of linear programming, the WLBP problem is equivalently transformed into a jointly constrained bilinear programming problem which is then reduced to a disjoint bilinear programming problem. Finally, in Theorem 4.2, we have shown that the resolution of this jointly constrained bilinear programming problem is equivalent to the resolution of a disjoint bilinear programming problem. Therefore, this may provide a possibility to solve the WLBP problem via a disjoint bilinear programming problem. For future research, it is interesting to discuss the weak nonlinear bilevel programming problems.

\section{Acknowledgments}

The authors are greatly indebted to the anonymous reviewers and editors for their valuable comments and insightful suggestions which greatly improved the previous version of the paper. The work presented in this paper was supported by the National Natural Science Foundation of China (Nos. 11501233, 71501023) and the Key Project of Anhui Province University Excellent Youth Support Plan (No. gxyqZD2016102).

\section{References}

[1] A. Aboussoror, A. Mansouri. Weak linear bilevel programming problems: existence of solutions via a penalty method. J. Math. Anal. Appl. 304 (2005)399-408.

[2] A. Aboussoror, A. Mansouri. Existence of solutions to weak nonlinear bilevel problems via MinSup and d.c. problems. RAIRO Oper. Res. 42(2008)87-103.

[3] A. Aboussoror, S. Adly, V. Jalby. Weak nonlinear bilevel problems: existence of solutions via reverse convex and convex maximization problems. J. Indus. Manage. Optim. 7(2011)559-571.

[4] S. Alarie, C. Audet, B. Jaumard, G. Savard. Concavity cuts for disjoint bilinear programming. Math. Program. 90(2) (2001) 373-398.

[5] F.A. Al-Khayyal. Jointly constrained bilinear programs and related problems: An overview. 
Comput. Math. Appl. 19 (1990) 53-62.

[6] F.A. Al-Khayyal. Generalized bilinear programming: part i. models, applications and linear programming relaxation. Eur. J. Oper. Res. 60(3) (1992) 306-314.

[7] C. Audet, P. Hansen, B. Jaumard, G. Savard. A symmetrical linear maxmin approach to disjoint bilinear programming. Math. Program. 85(1999)573-592.

[8] X.J. Ban, S. Lu, M. Ferris, H.X. Liu. Risk averse second best toll pricing. in Transportation and Traffic Theory 2009: Golden Jubilee, Springer US, 2009, pp. 197-218.

[9] J.F. Bard. Practical bilevel optimization: algorithms and applications. Kluwer Academic, Dordrecht, 1998.

[10] L. Brotcorne, M. Labbe, P. Marcotte, G. Savard. A bilevel model for toll optimization on a multicommodity transportation network. Transp. Sci. 35(4) (2001) 345-358.

[11] M. Cervinka, C. Matonoha, J.V. Outrata. On the computation of relaxed pessimistic solutions to MPECs. Optim. Methods Software 28(2013) 186-206.

[12] A. Charnes, W.W. Cooper, G.L. Thompson. Some properties of redundant constraints and extraneous variables in direct and dual linear programming problems. Oper. Res. 10(1962) 711-723.

[13] B. Colson, P. Marcotte, G. Savard. An overview of bilevel optimization. Ann. Oper. Res. 153 (2007) 235-256.

[14] S. Dassanayaka. Methods of variational analysis in pessimistic bilevel programming. Wayne State University, PhD Thesis, 2010.

[15] S. Dempe. Foundations of bilevel programming. Nonconvex Optimization and its Applications Series, Kluwer Academic, Dordrecht, 2002.

[16] S. Dempe. Annottated bibliography on bilevel programming and mathematical problems with equilibrium constraints. Optimization 52 (2003)333-359.

[17] S. Dempe, B.S. Mordukhovich, A.B. Zemkoho. Necessary optimality conditions in pessimistic bilevel programming. Optimization 63(2014)505-533.

[18] X. Ding, F. Al-Khayyal. Accelerating convergence of cutting plane algorithms for disjoint bilinear programming. J. Global Optim. 38(3) (2007) 421-436.

[19] S. Effati, A. Mansoori, M. Eshaghnezhad. An efficient projection neural network for solving bilinear programming problems. Neurocomputing 168 (2015)1188-1197. 
[20] J.E. Falk. A linear max-min problem. Math. Program. 5(1) (1973)169-188.

[21] G. Gallo, A. Ulkucu. Bilinear programming: an exact algorithm. Math. Program. 12(1) (1977) 173-194.

[22] Y. Gao, G. Zhang, J. Lu, H.M. Wee. Particle swarm optimization for bi-level pricing problems in supply chains. J. Global Optim. 51 (2011)245-254.

[23] X. Guo, T. Hu, T. Zhang, Y. Lv. Bilevel model for multi-reservoir operating policy in inter-basin water transfer-supply project. J. hydrol. 424(2012)252-263.

[24] R. Horst, H. Tuy. Global optimization: Deterministic approaches, Springer, 1996.

[25] R.R. Iyer, I.E. Grossmann. A bilevel decomposition algorithm for long-range planning of process networks. Ind. Eng. Chem. Res. 37(2) (1998)474-481.

[26] V.V. Kalashnikov, S. Dempe, G.A. Perez-Valdes, N.I. Kalashnykova, J.F. Camacho-Vallejo. Bilevel programming and applications. Math. Pro. Eng. (2014).

[27] H. Konno. A cutting plane algorithm for solving bilinear programs. Math. Program. 11(1) (1976) 14-27.

[28] M.B. Lignola, J. Morgan. Topological existence and stability for Stackelberg problems. J. Optim. Theory Appl. 84(1995)145-169.

[29] J. Liu, Y.X. Fan, Z. Chen, Y. Zheng. Pessimistic bilevel optimization: a survey. Int. J. Comput. Intell. Syst. 11 (2018) 725-736.

[30] P. Loridan, J. Morgan. New results on approximate solutions in two-level optimization. Optimization 20 (1989) 819-836.

[31] P. Loridan, J. Morgan. $\varepsilon$-regularized two-level optimization problems: approximation and existence results. in Proceeding of the Fifth French-German Optimization Conference, Springer Berlin Heidelberg, 1989, pp. 99-113.

[32] P. Loridan, J. Morgan. Weak via strong Stackelberg problem: New results. J. Global Optim. 8 (1996) 263-287.

[33] R. Lucchetti, F. Mignanego, G. Pieri. Existence theorems of equilibrium points in Stackelberg games with constraints. Optimization 18(1987)857-866.

[34] J. Lu, J. Han, Y. Hu, G. Zhang. Multilevel decision-making: a survey. Inf. Sc. 346-347 (2016) 463-487.

[35] P. Marcotte, G. Savard. Bilevel programming: a combinatorial perspective. Graph theory and 
combinatorial optimization, Springer US, 2005, pp. 191-217.

[36] A. Marhfour. Mixed solutions for weak Stackelberg problems: existence and stability results. J. Optim. Theory Appl. 105(2000)417-440.

[37] K. Ritter. A method for solving maximum-problems with a nonconcave quadratic objective function. Zeitschrift fur Wahrscheinlichkeitstheorie und verwandte Gebiete 4(4) (1966)340-351.

[38] R.T. Rockafellar. Convex Analysis. Princeton University Press, Princeton, 1970.

[39] S. Saranwong, C. Likasiri. Product distribution via a bi-level programming approach: algorithms and a case study in municipal waste system. Expert Syst. Appl. 44 (2016) 78-91.

[40] A. Tsoukalas, W. Wiesemann, B. Rustem. Global optimisation of pessimistic bi-level problems. in Lectures on Global Optimization P.M. Pardalos and T.F. Coleman, eds., Fields Institute Communications 55, Americal Mathematical Society, Providence, 2009, 215-243.

[41] H.D. Sherali, A. Alameddine. A new reformulation-linearization technique for bilinear programming problems. J. Global optim. 4 (1992) 379-410.

[42] H. Vaish, C.M. Shetty. The bilinear programming problem. Nav. Res. Logist. 23(2) (1976) 303-309.

[43] W. Wiesemann, A. Tsoukalas, P. Kleniati, B. Rustem. Pessimistic bi-level optimisation. SIAM J. Optim. 23 (2013)353-380.

[44] G. Zhang, Y. Gao, J. Lu. Competitive strategic bidding optimization in electricity markets using bilevel programming and swarm technique. IEEE Trans. Ind. Electron. 58(2011) 2138-2146.

[45] G. Zhang, J. Lu, Y. Gao. Multi-level decision making: models, methods and applications, Springer, Berlin, 2015.

[46] G. Zhang, J. Han, J. Lu. Fuzzy bi-level decision-making techniques: a survey. Int. J. Comput. Intel. Systems 9 (2016) 25-34.

[47] Y. Zheng, Z. Wan, K. Sun, T. Zhang. An exact penalty method for weak linear bilevel programming problem. J. Appl. Math. Comput. 42(2013)41-49.

[48] Y. Zheng, Z. Wan, S. Jia, G. Wang. A new method for strong-weak linear bilevel programming problem. J. Indus. Manage. Optim. 11 (2015)529-547.

[49] Y. Zheng, D. Fang, Z. Wan. A solution approach to the weak linear bilevel programming problems. Optimization 7(2016) 1437-1449. 